\title{
The immunoproteasome is induced by cytokines and regulates apoptosis in human islets
}

\author{
Morten Lundh',2,a, Marco Bugliani3,a, Tina Dahlby', Danny Hung-Chieh Chou², \\ Bridget Wagner', Seyed Mojtaba Ghiasi', Vincenzo De Tata', Zhifei Chen', \\ Marianne Nissan Lund1,4, Michael J Davies', Piero Marchetti3,b and \\ Thomas Mandrup-Poulsen $1, \mathrm{~b}$
}

'Department of Biomedical Sciences, University of Copenhagen, Copenhagen, Denmark ${ }^{2}$ Chemical Biology and Therapeutics Program, Broad Institute of Harvard and MIT, Boston, Massachusetts, USA ${ }^{3}$ Department of Clinical and Experimental Medicine, University of Pisa, Pisa, Italy ${ }^{4}$ Department of Food Science, University of Copenhagen, Copenhagen, Denmark a(M Lundh and M Bugliani contributed equally as co-first authors)

$\mathrm{b}(\mathrm{P}$ Marchetti and T Mandrup-Poulsen contributed equally as co-senior authors)

Correspondence should be addressed to T Mandrup-Poulsen Email tmpo@sund.ku.dk

\begin{abstract}
In addition to degrading misfolded and damaged proteins, the proteasome regulates the fate of cells in response to stress. The role of the proteasome in pro-inflammatory cytokine-induced human beta-cell apoptosis is unknown. Using INS-1, INS-1E and human islets exposed to combinations of IFN $\gamma, \mathrm{IL}-1 \beta$ and TNF $\alpha$ with or without addition of small molecules, we assessed the role of the immunoproteasome in pancreatic beta-cell demise. Here, we show that cytokines induce the expression and activity of the immunoproteasome in INS-1E cells and human islets. Cytokine-induced expression of immunoproteasome subunits, but not activity, depended upon histone deacetylase 3 activation. Inhibition of JAK1/STAT1 signaling did not affect proteasomal activity. Inhibition of the immuno-proteasome subunit PSMB8 aggravated cytokine-induced human betacell apoptosis while reducing intracellular levels of oxidized proteins in INS-1 cells. While cytokines increased total cellular NFкB subunit P50 and P52 levels and reduced the cytosolic NFKB subunit P65 and IאB levels, these effects were unaffected by PSMB8 inhibition. We conclude that beta cells upregulate immuno-proteasome expression and activity in response to IFN $\gamma$, likely as a protective response to confine inflammatory signaling.
\end{abstract}

\author{
Key Words \\ - diabetes \\ - islet cells \\ - cytokines \\ inflammatory diseases
}

\section{Introduction}

The proteasome is responsible for cellular protein degradation, but in addition to eliminating damaged or misfolded proteins targeted for breakdown by poly-ubiquitination, the proteasome also regulates key cellular processes such as proliferation, growth, differentiation, gene transcription, signaling and apoptosis by modulating the half-life of cell cycle regulators and the processing of transcriptional activators and repressors (Bhattacharyya et al. 2014).

Proteasomal activity generates peptide fragments of exogenous or endogenous proteins that are complexed to major histocompatibility complex class I molecules 
for presentation to CD8+ T-cells, with this process being critical for adaptive immunity, immune surveillance and immune tolerance (Ferrington \& Gregerson 2012). By processing the p105 and p100 subunits of the master inflammatory transcription factor $\mathrm{NF \kappa B}$ to their p50 and p52 forms, the proteasome is also a key regulator of innate immunity and inflammation (Rape \& Jentsch 2002). These functions are executed by the so-called immunoproteasome (i-proteasome) consisting of the $20 \mathrm{~S}$ core particle in which the catalytic $\beta 1,2$ and 5 constitutive subunits (also known as Psmb6/LMP19, Psmb7/LMP9 and Psmb5/LMP17, respectively) are substituted with the cytokine-inducible $\beta 1 \mathrm{i}, 2 \mathrm{i}$ and $5 \mathrm{i}$ subunits (also termed Psmb9/LMP2, Psmb10/MECL-1 and Psmb8/ LMP7, respectively) (Ferrington \& Gregerson 2012). The i-proteasome lid function is fulfilled by complexing with the $11 \mathrm{~S}$ regulatory heptameric particle. Since i-proteasome processes smaller proteins and peptides, it is ubiquitin independent and does not possess ATPase activity (Ferrington \& Gregerson 2012). An intermediary proteasome composed of a mixture of subunits from the s- and i-proteasomes, and a thymus-specific proteasome with a $\beta 5 t$ for $\beta 5$ substitution, also exists.

Emerging evidence supports the hypothesis that the s-proteasome has important functions in pancreatic beta cells (Hartley et al. 2009). The s-proteasome balances betacell proinsulin levels (Kitiphongspattana et al. 2005) and K/ATP channel expression (Yan et al. 2005), and degrades Ucp2 and 3 proteins, improving glucose-stimulated insulin secretion (Brand et al. 2010). The s-proteasome protects beta cells from lipotoxic ER stress and maintains an antiapoptotic Bcl2 protein family balance (Litwak et al. 2015). Glucotoxicity inhibits s-proteasome activity leading to caspase-3-dependent apoptosis in INS-1E cells and human islets, an effect mimicked by s-proteasome inhibition (Broca et al. 2014).

Although the proteasome degrades the interleukin-1 receptor-activated kinase IRAK after IL-1-induced phosphorylation (Yamin \& Miller 1997), inhibition of the s-proteasome reduces IL-1-induced beta-cell iNOS expression, NO synthesis and PGE2 production, possibly by preventing s-proteasome-dependent activation of the NFkB pathway (Kwon et al. 1998). Accordingly, s-proteasome inhibition modestly decreases cytokine-induced islet toxicity, while suppressing islet insulin release (Storling et al. 2005).

The function(s) of the i- or intermediate proteasomes in beta cells are poorly understood. The $11 \mathrm{~S}$ regulatory particle of the i-proteasome has been reported to mediate
MafA degradation leading to impaired beta-cell function (Kanai et al. 2011). However, the role of the i-proteosome in inflammatory beta-cell dysfunction or viability is unknown. Here, we hypothesized that the expression of the s-and i-proteasome subunits are differentially regulated by inflammatory cytokines and that inhibition of i-proteasome activity affects cytokine-induced beta-cell apoptosis.

\section{Materials and methods}

\section{Cell culture and reagents}

INS-1 and INS-1E cells (generously provided by Claes Wollheim and Pierre Maechler, University of Geneva, Switzerland) were maintained as previously described (Storling et al. 2005, Chou et al. 2012). Cells were mycoplasma negative. Human pancreatic islets were isolated by collagenase digestion, density gradient purification and handpicking from the glands of 16 organ donors (for donor characteristics, Table 1) and then cultured in M199 culture medium, supplemented with $10 \%$ bovine serum and antibiotics in a $\mathrm{CO}_{2}$ incubator at $37^{\circ} \mathrm{C}$ as described previously (Bugliani et al. 2013). No gender-related differences were noted for any outcome variable, and data are therefore combined.

Recombinant rat (rr) IL-1 $\beta$ and recombinant mouse (rm) TNF $\alpha$ were purchased from R\&D Systems. Rm IFN $\gamma$ and Griess reagent were purchased from Sigma. Recombinant human (rh) IL-1 $\beta$ and IFN $\gamma$ were purchased from Roche Diagnostics. Trypsin-, chymotrypsin- and caspase-like proteasomal activity reagents were purchased from Promega. The broad proteasome inhibitor MG132 and the selective immunoproteasome inhibitor ONX-914 (200 nM), 20- to 40-fold more selective for Psmb8 than Psmb9 (Muchamuel et al. 2009), were from Selleckchem (Houston, Texas, USA). The histone deacetylase (HDAC)1-3 inhibitor MS-275 was from Selleckchem, whereas the inhibitor of the deubiquitinase ubiquitinspecific peptidase 9X (USP9X) BRD0476 that in turn highly selectively inhibits Janus-activated kinase (JAK)/ signal transducer and activator of transcription (STAT) 1 and the HDAC1-2 selective ' 3 ' inhibitor were synthesized in-house (Chou et al. 2012, 2015).

\section{Gene profiling}

INS-1E cells were exposed to rrIL-1 $\beta(10 \mathrm{ng} / \mathrm{mL}), \mathrm{rmTNF} \alpha$ $(25 \mathrm{ng} / \mathrm{mL})$ and $\mathrm{rmIFN} \gamma(50 \mathrm{ng} / \mathrm{mL}) \pm \mathrm{MS} 275$ or ' 3 ' $(5 \mu \mathrm{M})$

Published by Bioscientifica Ltd 
Table 1 Characteristics of the 16 human donor and islet preparations.

\begin{tabular}{lc}
\hline Mean age \pm s.D. (years) & $62 \pm 18$ \\
Gender M/F & $7 / 9$ \\
Mean BMI \pm s.D. $\left(\mathrm{kg} / \mathrm{m}^{2}\right)$ & $26.0 \pm 2.7$ \\
Cause of death & 14 CVD*/2 trauma \\
Mean ICU stay \pm s.D. (days) & $4 \pm 3$ \\
Mean plasma glucose levels during ICU & $149 \pm 24$ \\
$\quad$ stay \pm S.D. (mg/dL) & $15 \pm 2$ \\
Mean pancreas cold ischemia time \pm s.D. (h) & $69 \pm 22 \%$ \\
Mean islet preparation purity** \pm S.D. & $2.9 \pm 1.0$ \\
Stimulated insulin release/basal & \\
insulin release \pm S.D. &
\end{tabular}

*CVD cardio-/cerebrovascular disease; **evaluated by dithizone staining.

for $6 \mathrm{~h}$. Gene expressions (triplicates) were analyzed using the Affymetrix rat 230.2 array (Genomic Services, Broad Institute, MA, USA) and regulated gene sets were determined using the Gene Set Enrichment Analysis (GSEA) method (Subramanian et al. 2005).

\section{qPCR}

INS-1E cells were exposed to recombinant rodent cytokines \pm MS-275 or BRD0476 $(10 \mu \mathrm{M})$ for the indicated time points. Gene expressions were determined using SYBR green (Applied Biosystems) with selective primer sets. Hprt1 was used as reference gene. Human islets were exposed from 6 to $24 \mathrm{~h}$ to $50 \mathrm{U} / \mathrm{mL}$ ( $1 \mathrm{ng} / \mathrm{mL}$ ) rhIL$1 \beta$ and/or $1000 \mathrm{U} / \mathrm{mL}(50 \mathrm{ng} / \mathrm{mL}) \mathrm{rhIFN}-\gamma$. At the end of the incubation period, total RNA was extracted using the PureLink RNA Mini Kit (Life Technologies) and quantified by absorbance at A260/A280 nm (ratio >1.9) in a NanoDrop 2000C spectrophotometer (Euroclone Spa, Pero, Italy). For quantitative real-time PCR experiments, $1 \mu \mathrm{g}$ total RNA was reversely transcribed using a SuperScript VILO cDNA Synthesis Kit (Life Technologies). The primers of interest were obtained from assay-on-demand gene expression products (Life Technologies). mRNA levels were quantified and normalized for $\beta$-actin using a ViiA 7analyser (Life Technologies). Rat primer sequences are listed in Table 2.

Table 2 Rat primer sequences.

\begin{tabular}{|c|c|c|}
\hline Target & Forward primer & Reverse primer \\
\hline Psma2 & CATTCAGCCCATCTGGTAAA & GGTTATGGGCTCCACTTTG \\
\hline Psma7 & CAGTCAGGTGGCAAAAACAT & ACTTAGAATCTTGAGGGGCTGA \\
\hline Psmb10 & GAATGCGTCCTTGGAACAC & AGCCCCACAGCAGTAGATTT \\
\hline Pmse1 & AGCCAACCTGAGCAATCTG & TCCTTCTCCTGTTGCTTCTTC \\
\hline Hprt1 & GCAGACTTTGCTTTCCTTGG & CCGCTGTCTTTTAGGCTTTG \\
\hline
\end{tabular}

\section{Western blotting}

INS-1E cells were exposed to $1 \mathrm{ng} / \mathrm{mL}$ IL- $1 \beta$ and $50 \mathrm{ng} / \mathrm{mL}$ IFN $\gamma$ for $30 \mathrm{~min}$ or $6 \mathrm{~h}$ as indicated in figure legends with or without $200 \mathrm{nM}$ of ONX-914. Cells were lysed in RIPA buffer (for NFkB subunits p50 and p52), and cytosolic extracts (for p65) were prepared according to the protocol from Abcam: http://www.abcam.com/ps/pdf/ protocols/subcellular_fractionation.pdf. Western blots were performed using antibodies against 46 and $54 \mathrm{kDa}$ phosphor-JNK isoforms, p50, p52, p65, IкB or $\beta$-actin $/ \beta / \alpha$ tubulin (reference protein). Primary antibodies: pJNK 1:1000 (Cell Signaling), IkBa 1:200, cat.no: sc-371 (Santa Cruz Biotechnology), 1:4000, cat.no: T6074 (SigmaAldrich). Secondary antibodies: 1:5000 anti-rabbit IgG, cat.no: 7074S (Cell Signaling), 1:5000 anti-mouse IgG, cat.no: 7076S (Cell Signaling).

\section{Proteasomal activity}

INS-1E cells were exposed to IL- $1 \beta+\mathrm{TNF} \alpha+\mathrm{IFN} \gamma$ cytokine combination \pm MS275 $(5 \mu \mathrm{M})$ or BRD0476 $(10 \mu \mathrm{M})$, while human islets were exposed to the IL- $1+$ IFN cytokine combination or individual cytokines for the indicated times. Trypsin-, chymotrypsin- and caspase-like proteasomal activities were determined by a luminescent assay (Promega). The proteasome inhibitor MG132 $(0.5 \mu \mathrm{M})$ was included as a positive control.

\section{Electron microscopy analysis}

Quantification of apoptotic beta cells was performed as previously detailed (Masini et al. 2009). Beta cells were identified based on the presence of typical beta granules, while apoptotic beta cells were identified based on the appearance of marked chromatin condensation and/or blebs, as previously reported (Masini et al. 2009). Beta cells (400-450 per experimental condition) were counted by a blinded observer, from a total 7 islets from two separate islet preparations.

\section{Markers of protein oxidation}

INS- 1 cells were exposed to $150 \mathrm{pg} / \mathrm{mL}$ IL- $1 \beta+0.1 \mathrm{ng} / \mathrm{mL}$ IFN $\gamma$ for $6,12,18$ and $24 \mathrm{~h}(n=6)$. The cells were then rinsed three times with cold PBS and re-suspended in cold PBS with $10 \mu \mathrm{g} / \mathrm{mL}$ protease/phosphatase inhibitor (Sigma-Aldrich) plus $5 \mathrm{mM}$ EDTA. The cells were lysed on ice by ultra-sonication (10 s on/off cycles for $5 \mathrm{~min}$ ) and spun down $\left(20,000 \mathrm{rcf}, 4^{\circ} \mathrm{C}, 5 \mathrm{~min}\right)$. Protein concentrations

Published by Bioscientifica Ltd 
were determined by the Bradford reagent using $1 \mu \mathrm{L}$ of lysate in triplicate. A BSA standard curve was included for quantification. Lysate concentrations of protein carbonyls and thiols, as markers for protein oxidation, were determined using an OxiSelect protein carbonyl ELISA kit (Cell Biolabs Inc, San Diego, USA) according to the manufacturer's instructions with samples adjusted to $0.01 \mathrm{mg} / \mathrm{mL}$ protein, and by reaction of $0.20 \mathrm{mg} / \mathrm{mL}$ protein with 5,5'-dithiobis-(2-nitrobenzoic acid) (DTNB) (Hawkins et al. 2009), respectively. In order to have all samples measured on single microplates, only the first five batches were analyzed for protein carbonyls. Sodium hydrogen phosphate, sodium dihydrogen phosphate, GSH, urea and ethanol were purchased from SigmaAldrich. Phosphate-buffered saline (PBS) $20 \times$ concentrate (pH 7.5) was obtained from Amresco (Solon, OH, USA).

\section{Statistics}

Data were analyzed using GraphPad Prism or SAS 9.4 (SAS Institute Inc., Cary, NC, USA) using analysis of variance (ANOVA) followed by Bonferroni post hoc tests corrected for multiple comparisons where appropriate. $P<0.05$ was considered significant. For human islet, data are presented as means \pm S.D. Difference between two groups was assessed by two-tailed Student's $t$-test, or by ANOVA for multi-group comparisons. Differences between protein oxidation marker levels were compared by contrast analysis (ls means).

\section{Results}

\section{Proinflammatory cytokines induce the expression of proteasome-coding genes}

To uncover novel pathways involved in cytokine-mediated beta-cell apoptosis, we undertook an unbiased approach by performing a microarray-based gene expression analysis on INS-1E cells exposed to the cytokines IL-1 + TNF + IFN for $6 \mathrm{~h}$. Enriched gene sets were identified, and among the top scoring sets was the cluster annotated as 'REACTOME_STABILIZATION_OF_P53' which consists of genes encoding the proteasome (Fig. 1A).

Heat plot analysis of this gene set revealed differential regulation of the inflammasome components by cytokines. The $20 \mathrm{~S}$ Psmb 3, 4, 5 and 6 catalytic (protease) subunits (also known as $\beta 3, \beta 7$, $\beta 5$ and $\beta 1$, respectively), the 19S Psmc1, 2, 5 and 6 regulatory ATPase subunits (also known as Rpt2, Rpt1,
Rpt6 and Rpt4, respectively), the 19S Psmd 2, 6 and 8 non-ATPase regulatory subunits (also known as Rpn1, Rpn7 and Rpn12, respectively) as well as ubiquitin B were slightly to moderately downregulated by the cytokine combination (red $\rightarrow$ pink). In contrast, the $20 \mathrm{~S}$ Psmb1, 2, 7, 8, 9 and 10 catalytic subunits (also known as $\beta 6, \beta 4, \beta 2, \beta 5 \mathrm{i}, \beta 1 \mathrm{i}$ and $\beta 2 \mathrm{i}$, respectively), the $20 \mathrm{~S}$ Psma $1,2,4,5,6$ and 7 structural subunits (also known as $\alpha 6$, $\alpha 2, \alpha 3, \alpha 5, \alpha 1$ and $\alpha 4$, respectively) and the 11S Psme 1 and 2 activator subunits (also known as $11 \mathrm{~S} \alpha$ and $11 S \beta$, respectively) were upregulated by the cytokine combination (blue $\rightarrow$ red). Since Psmb 8, 9 and 10 are inducible components of the immunoproteasome, and since the function of this proteasome is ubiquitin independent and thus also 19 proteasome ATPase activity independent (Ferrington \& Gregerson 2012), this microarray expressional pattern is consistent with a role for inflammatory cytokines in upregulating the immunoproteasome in insulin-producing cells.

Four of the cytokine-inducible genes (Psma2, Psma7, Psme1 and Psmb10) were selected on the basis of their pronounced regulation and functions in the 19S, 20S and $11 \mathrm{~S}$ proteasome particles (Fig. 1A) for qPCR validation. We confirmed that cytokine induced the expression of these genes after $6 \mathrm{~h}$ (Fig. 1B, C, D and E). Induction of Psma7, Psme1 and Psmb10 was also confirmed at later time points (Fig. 1C, D and E).

In human islets, exposure to the cytokine combination IL-1+IFN induced a significant increase in the expression of the immunoproteasomal subunits Psmb8, Psmb9 and Psmb10; however, the expression of the constitutive Psmb5, Psmb6 and Psmb7 proteasome components (Fig. 2) was not altered. A similar induction was observed in human islets exposed for $6 \mathrm{~h}$ to IFN- $\gamma$, but not IL- $1 \beta$ (Fig. 2), consistent with the presence of an IFN- $\gamma$-inducible immunoproteasome in pancreatic islets.

\section{Proinflammatory cytokines induce proteasomal activity in human islets}

We next assessed the effect of cytokines on proteasomal catalytic activity. Human islets were exposed for $6-24 \mathrm{~h}$ to the IL- $1 \beta+$ IFN- $\gamma$ cytokine combination or individual cytokines. Trypsin-, chymotrypsin- or caspase-like activities, which are shared between the constitutive proteasome and the immunoproteasome, were then quantified. Twenty-four-hour exposure to the cytokine combination induced a significant increase in all three http://joe.endocrinology-journals.org DOI: $10.1530 / J O E-17-0110$ (c) 2017 Society for Endocrinology Printed in Great Britain
Published by Bioscientifica Ltd. 
A

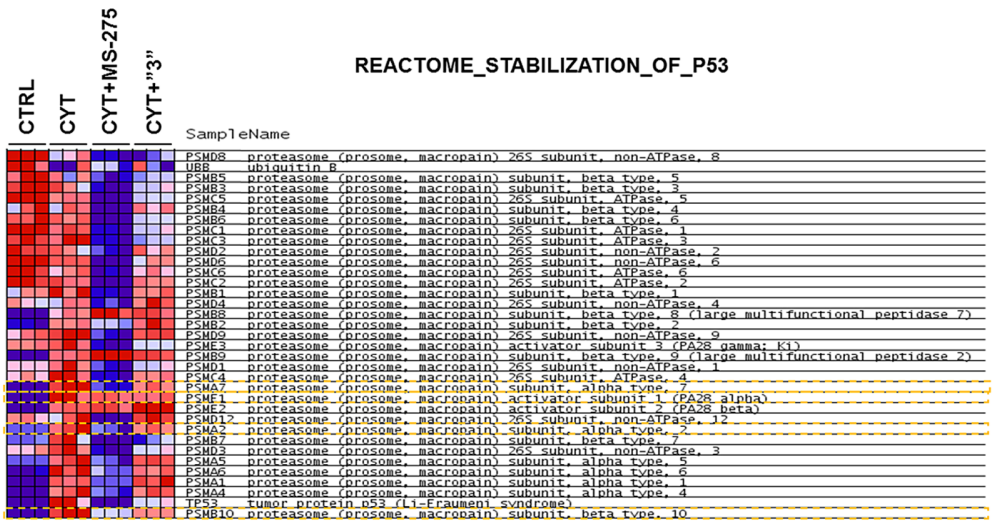

B
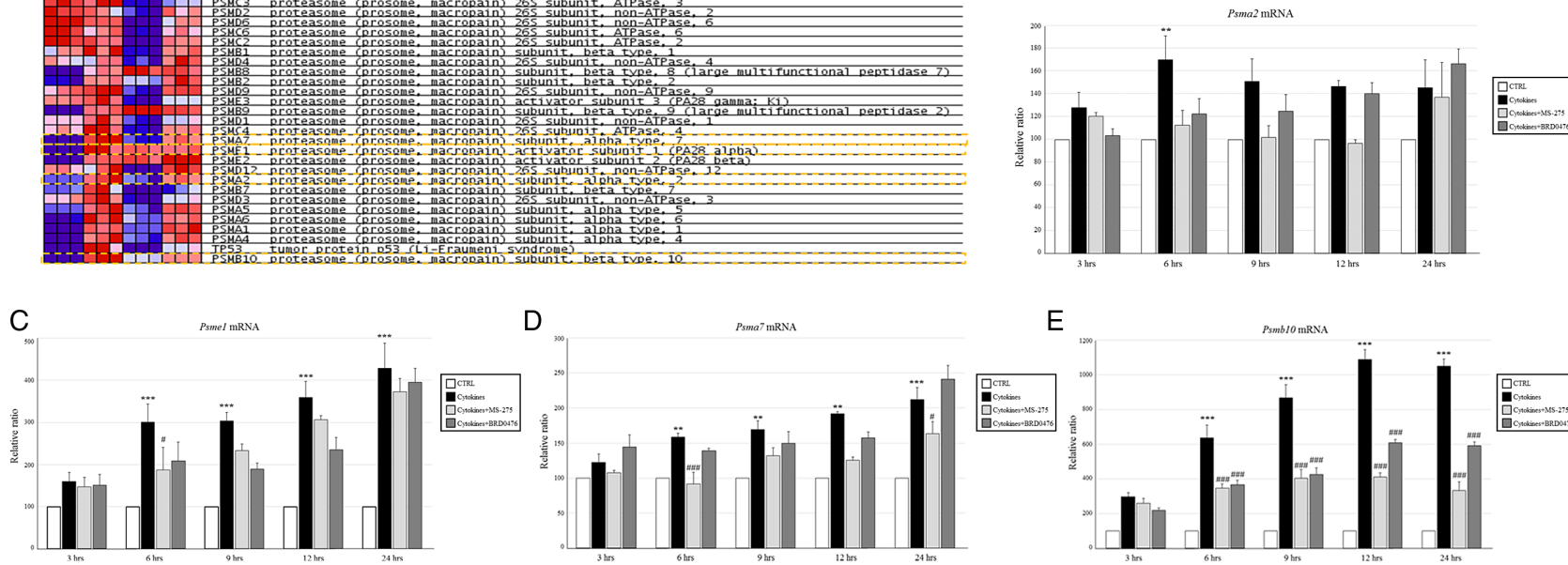

D

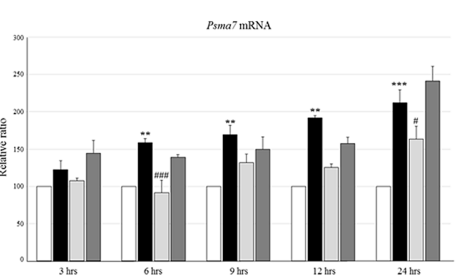

E

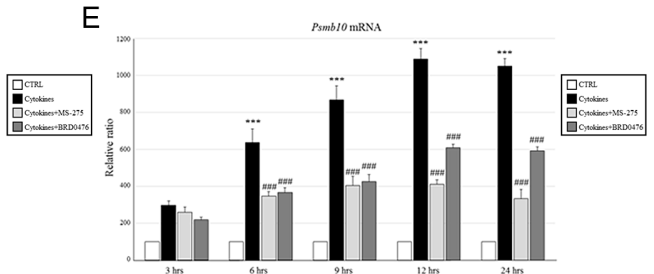

Figure 1

Gene set enrichment analysis (GSEA) and validation of selected genes in INS-1E cells exposed to cytokines and HDAC inhibitors. (A) INS-1E cells were exposed to cytokines (IL-1 $\beta+\mathrm{TNF} \alpha+\mathrm{IFN} \gamma) \pm$ the HDAC1-3 selective inhibitor MS-275 or the HDAC1-2 selective inhibitor ' 3 ' for $6 \mathrm{~h}$. RNA was purified, and a microarray followed by GSEA was performed. The top scoring gene set is shown here. Selected gene expressions are highlighted in yellow, $n=1$.

(B, C, D and E) INS-1E cells were exposed to vehicle (white bars), cytokines (black bars) \pm MS-275 (light grey bars) or BRD0476 (dark grey bars) for 3, 6, 9, 12 or $24 \mathrm{~h}$. Expression of (B) Psma2, (C) Psme1, (D) Psma7, (E) Psmb10 was analyzed by qPCR. Results are shown as means + s.E.M., $n=4$. ${ }^{*} P<0.05, * * P<0.01$ and $* * * P<0.001$ vs vehicle (CTRL), $\# P<0.05, \# \# P<0.001$ vs cytokines.

activities, with the caspase-like activity being the least upregulated (Fig. 3A). In accordance with the finding that IFN $\gamma$ was responsible for the transcriptional regulation of the inducible immunoproteasome components (Fig. 2), this effect was replicated by 6-h exposure to IFN- $\gamma$ alone (Fig. 3B) with a preponderance of chymotrypsin-like activity. Of note, TNF alone did not induce any expression

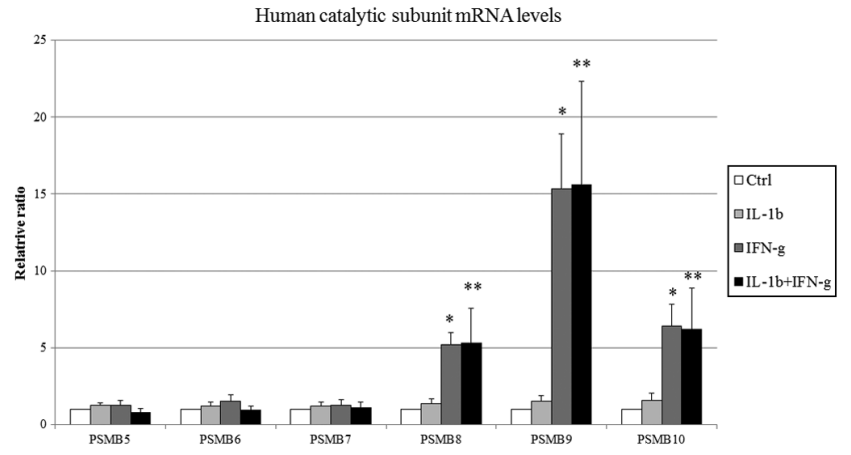

Figure 2

Expression of selected proteasomal subunit genes in human islets exposed to cytokines. The expression of PSMB5, PSMB6, PSMB7, PSMB8, $P S M B 9$ and $P S M B 10$ was examined in human islets exposed to $(A)$ a combination of IL- $1 \beta$ and IFN $\gamma$ (black bars) or (B) only IL-1 $\beta$ (grey bars) or IFN $\gamma$ (black bars). Results are shown as means + s.E.M., $n=4-8$. ${ }^{*} P<0.05$ and $* * P<0.01$ vs other groups. of the selected immunoproteasomal subunits, nor did it increase proteasomal activities (data not shown). We also investigated the effect of high glucose on human islet proteasomal activity; 24-h exposure to $22.2 \mathrm{mM}$ glucose caused an increase in $\sim 40 \%$ trypsin-like activity $(P<0.05$, data not shown).

\section{Effects of selective immunoproteasome inhibition on human beta-cell death}

To understand whether immunoproteasomal activity mediates cytokine-induced beta-cell apoptosis, human islets were exposed for $24 \mathrm{~h}$ to IL- $1 \beta+\mathrm{IFN}-\gamma$ in the presence or absence of ONX-914, a potent and selective immunoproteasome inhibitor, with minimal crossreactivity with the s-proteasome. As shown in Fig. 4A, these cytokines significantly increased the proportion of apoptotic human islet cells, as identified by the classical morphological criteria of chromatin condensation and/or blebs (Fig. 4B). This effect was exacerbated by concomitant exposure to ONX-914, suggesting that cytokine-inducible immunoproteasomal activity is a protective response against inflammatory stress.

Published by Bioscientifica Ltd. 
A
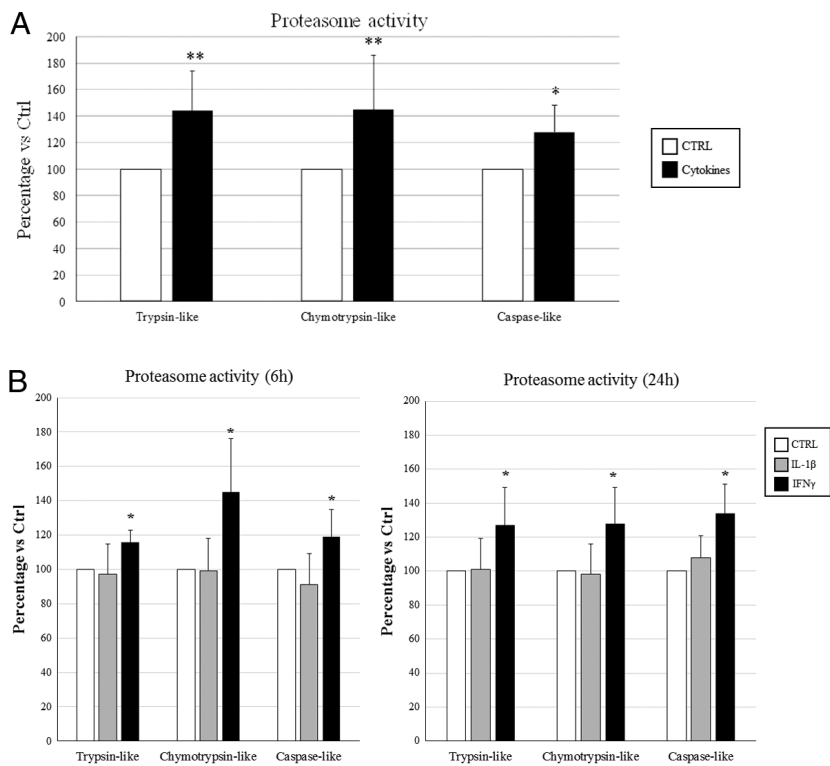

Figure 3

Proteasomal activities in human islets exposed to cytokines. The trypsin-like, chymotrypsin-like and caspase-like proteasomal activities were analyzed in human islets exposed to $(A)$ a combination of IL-1 $\beta$ and IFN $\gamma$ (black bars) or (B) only IL-1 $\beta$ (grey bars) or IFN $\gamma$ (black bars). Results are shown as means + S.E.M., $n=4-7$. ${ }^{*} P<0.05$ and $* * P<0.01$ vs other groups.

Proteasomal gene expression, but not activity, is reduced by small molecule inhibitors of HDACs and the JAK1/STAT pathway

We have shown that the deleterious effects of cytokines on beta cells can be prevented with small molecule blockade of the NFkB pathway by HDAC inhibition (Christensen et al. 2014), or the JAK-STAT1 pathway (Chou et al. 2015). We therefore examined whether cytokine-induced expression of proteasomal genes was affected similarly. MS-275 (an HDAC1-3 inhibitor) reduced several of the cytokineinduced genes of the GSEA, an effect not mimicked by an HDAC1-2 inhibitor (Chou et al. 2012) (Fig. 1A), suggesting that HDAC3 is a major regulator of the beta-cell immunoproteasome. Notably, however, cytokine-induced upregulation of the immunoproteasome components Psmb8 and Psmb9 and the $11 \mathrm{~S}$ activator subunits Psme1 and Pmse 2 were not counteracted by HDAC or STAT1 inhibition, suggesting that key components of the immunoproteasome were regulated by NFkB- and JAK/ STAT1-independent pathways.

We validated the microarray findings by qPCR and confirmed the activity of MS-275 in reducing the expression of Psma7 after 6h, and Psmb10 at all time points (Fig. 1D and E). In line with the microarray data, MS-275 did not reduce Psma2 expression (Fig. 1B) and only significantly reduced Psme1 after $6 \mathrm{~h}$ (Fig. 1C). Inhibition of the JAK1-STAT1 pathway by the ubiquitin-specific peptidase 9X inhibitor BRD0476 (Chou et al. 2015) only reduced the expression of Psmb10 (Fig. 1E), suggesting that the JAK2-activated transcription factor ATF2 contributes to the IFN $\gamma$-mediated immunoproteasome gene expression as shown in Fig. 2.

Since these small molecule inhibitors reduced the expression of selected proteasomal genes, we investigated the ability of the compounds to reduce proteasomal activity. Cytokine exposure increased the proteasomal
A

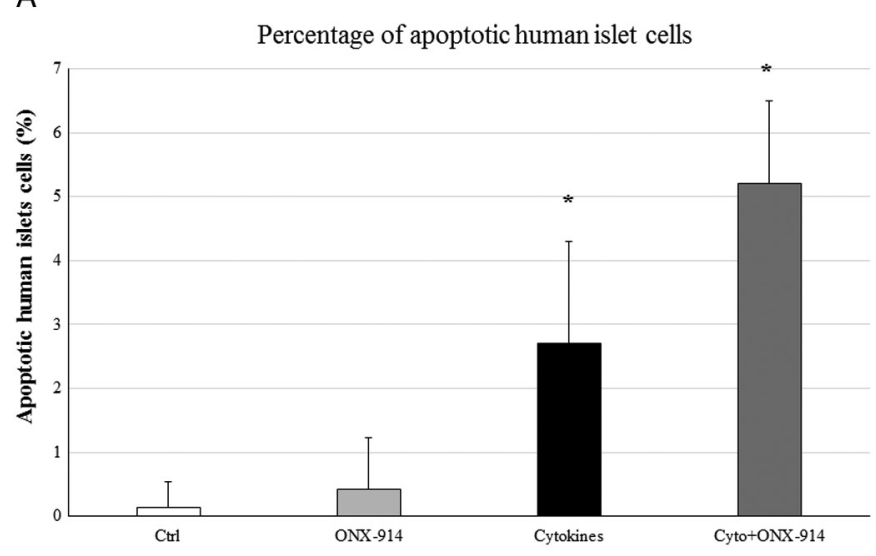

$\mathrm{B}$

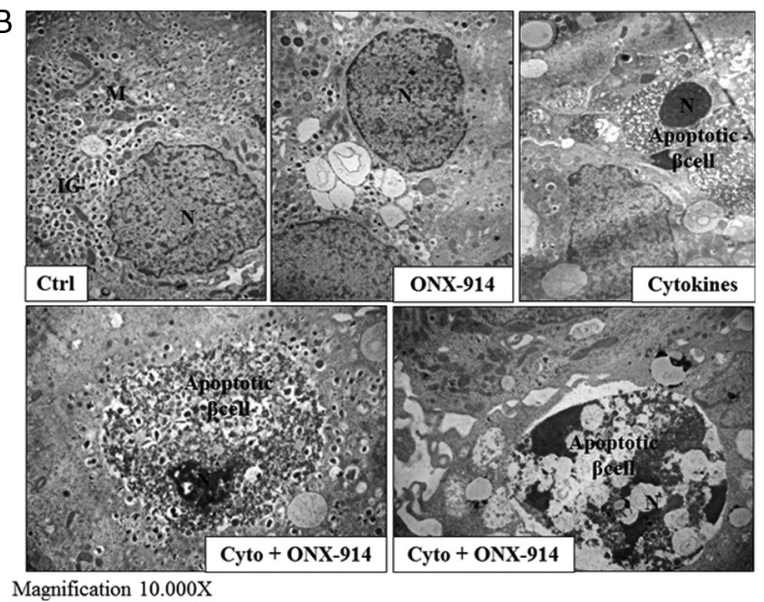

\section{Figure 4}

Cytokine-induced apoptosis is exacerbated by inhibition of the proteasome in human islets. Quantification of apoptotic human islet beta cells (A), identified by chromatin condensation and/or blebs in cells containing typical $\beta$ granules by transmission electron microscopy analysis of human islets (B). The quantified analysis in panel A depicts data as means +s.E.M. for islets exposed to vehicle (white bar), ONX-914 (light grey bar), cytokines (black bar) or cytokines + ONX-914 (dark grey bar); $n=7, * P<0.05$ vs other groups. 

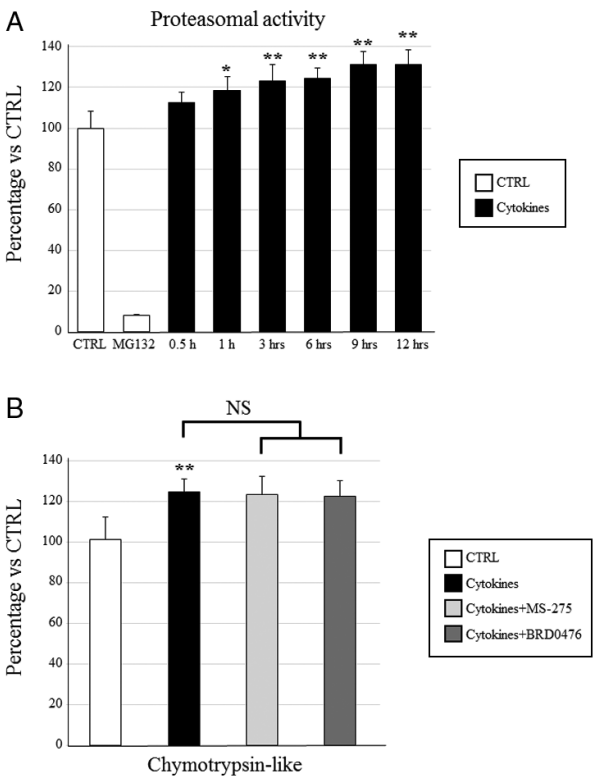

Figure 5

Proteasomal activity of INS-1E cells exposed to cytokines and small molecule inhibitors of cytokine-induced apoptosis. Chymotrypsin-like proteasomal activity was measured in (A) a time-dependent manner and (B) in INS-1E cells exposed for cytokines \pm MS-275/BRD0476 for $12 \mathrm{~h}$. The broad proteasome inhibitor MG-132 was used as a positive control. (A) Eleven independent wells, (B) twenty-two independent wells, results are shown as means + STD. ${ }^{*} P<0.01$ and ${ }^{*} P<0.001$ vs CTRL, NS, no significance.

activity in a time-dependent manner (Fig. 5A), in line with our human islet data, suggesting a relationship of this phenomenon to the beta-cell compartment of the human islet (Fig. 4). Interestingly, none of the small molecule inhibitors affected cytokine-induced activity (Fig. 5B), indicating that the inhibition of proteasomal activity does not contribute to HDAC inhibition-mediated protection against inflammatory beta-cell stress, and that beta-cell proteasomal activation by cytokines was JAK1/STAT1- and ubiquitin independent.

\section{Markers of protein oxidation}

We next examined the mechanism underlying the i-proteasome inhibition-mediated aggravation of cytokine-induced beta-cell apoptosis. The i-proteasome plays an important role in clearing oxidatively damaged proteins generated as a consequence of inflammation, thereby preventing protein aggregation (Seifert et al. 2010) and the triggering of apoptosis (Chang \& Chang 2015). We therefore quantified the depletion of protein thiols and formation of protein carbonyls that can be generated on a broad range of amino acids by oxidation. Cytokines did not

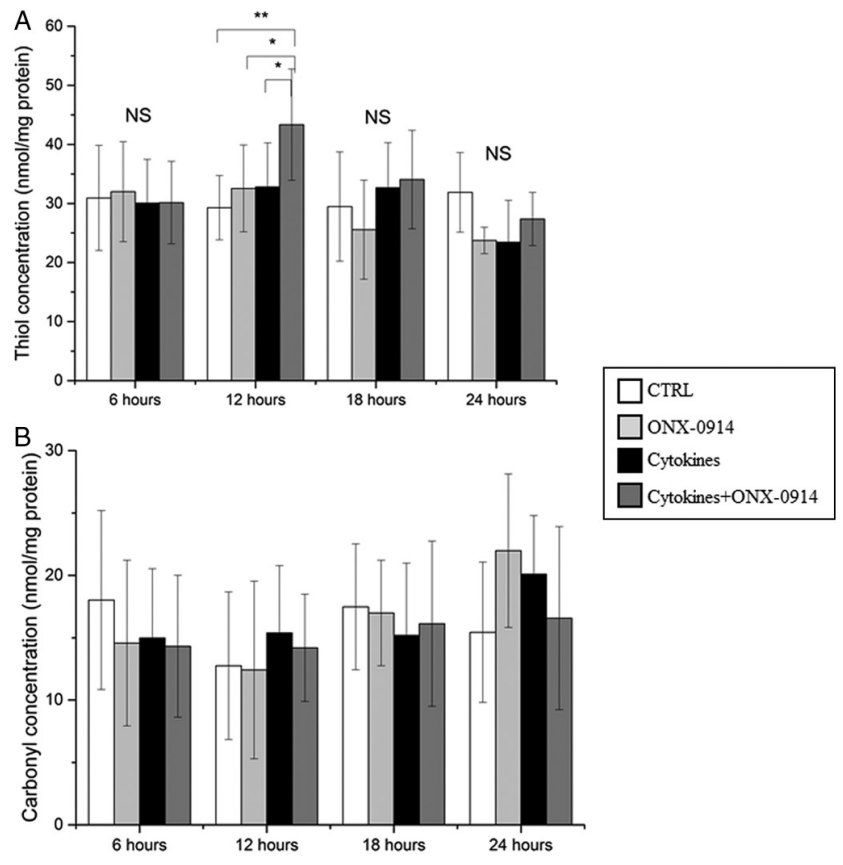

Figure 6

I-proteasome inhibition does not cause accumulation of oxidized proteins in cytokine-exposed INS-1 cells. (A) Thiol concentration in INS-1 cell lysates incubated with cytokines (Cyt), $200 \mathrm{nM}$ Psmb8 inhibitor ONX-914 (CtI/ONX) or both (Cyt/ONX) for $6,12,18$ or $24 \mathrm{~h}$. Data are presented as mean \pm S.D., $n=6$. (B) Carbonyl concentration in INS-1 cell lysates incubated with cytokines (Cyt), Psmb8 inhibitor (CtI/ONX) or both (Cyt/ONX) for $6,12,18$ or $24 \mathrm{~h}$. Data are presented as mean \pm s.D., $n=5$. Two-way ANOVA: $P=0.0275$; One-way ANOVAs: $6 \mathrm{~h} P=0.9786 ; 12 \mathrm{~h}$ $P=0.0421 ; 18 \mathrm{~h} P=0.4129 ; 24 \mathrm{~h} P=0.0835$. Ad hoc Student's $t$-tests for $12 \mathrm{~h}$ : $* P<0.05, * * P<0.01$

alter oxidized protein levels in the presence of a functional proteasome (Fig. 6). I-proteasome inhibition did not cause an accumulation, but in fact a reduction of oxidized proteins in the presence of cytokines, as evidenced by an increase in thiol levels (Fig. 6A). Protein carbonyls were not affected by i-proteasomal inhibition (Fig. 6B). These data indicate that accumulation of damaged proteins is not the mechanism of action underlying ONX-914-mediated aggravation of inflammatory beta-cell apoptosis.

\section{Effects of i-proteasome inhibition on beta-cell inflammatory signaling}

Since the i-proteasome regulates the activity of the $\mathrm{NF \kappa B}$ and MAPK pathways (Rape \& Jentsch 2002) we next asked if i-proteasomal inhibition affected the processing of p105/100 or degradation of p65 and ІкB, or altered JNK activity in INS-1E cells. Cytokines increased total cellular NFkB subunit p50 and p52 levels and reduced 
A $\quad$ p50
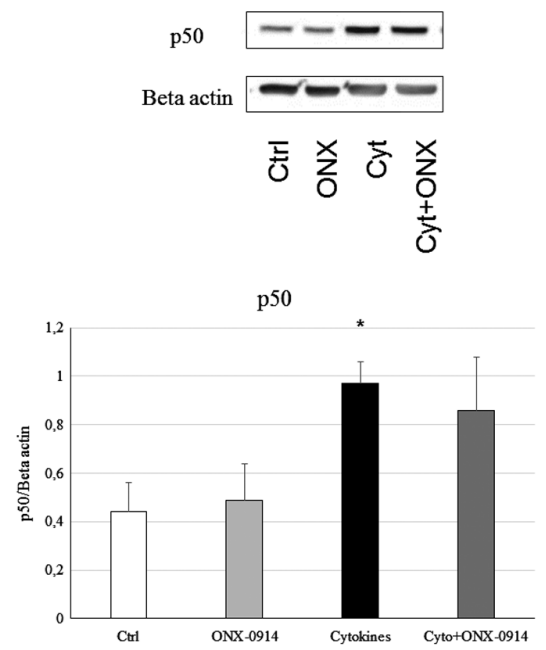

B NFKB p52

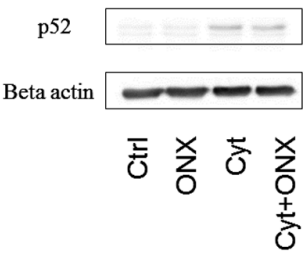

p52

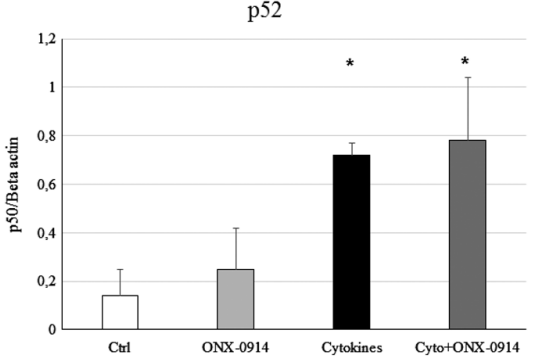

C
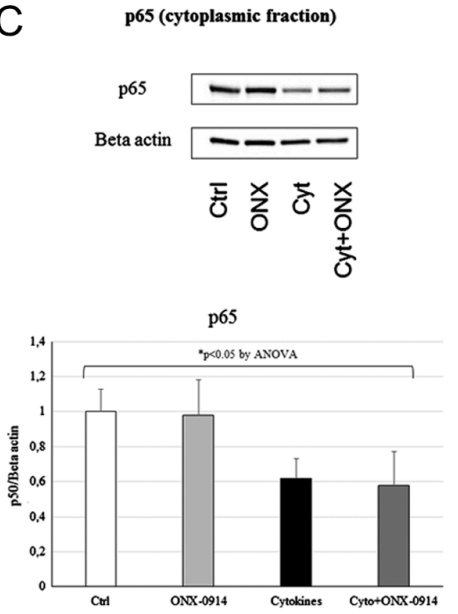

D

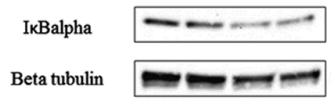

要希范希

I $\kappa$ B alpha

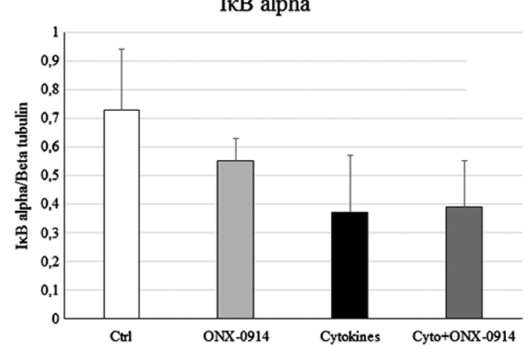

E

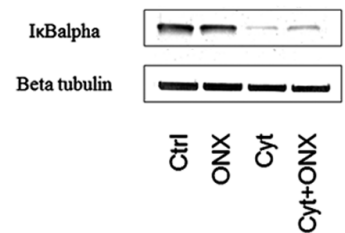

Figure 7

(A, B, C and D) INS-1E cells were exposed to $1 \mathrm{ng} / \mathrm{mL}$ IL-1 $\beta$ plus $50 \mathrm{ng} / \mathrm{mL}$ IFN $\gamma$ for $6 \mathrm{~h}$ without or with ONX-914 (200 $\mathrm{nM}$ ). Western blots were performed using anti-p50, p52, IкB alpha or p65 as well as anti-beta actin and anti-tubulin antibodies. For p65 analysis (C), cytoplasmic fractions were analyzed. (E) INS-1 cells were exposed to $1 \mathrm{ng} / \mathrm{mL}$ IL-1 $\beta$ plus $50 \mathrm{ng} / \mathrm{mL}$ IFN $\gamma$ for $30 \mathrm{~min}$ without or with ONX-914 (200 nM). Western blots were performed using IKB alpha and anti-tubulin antibodies. Data are expressed as ratios to house-keeping protein in percentage of control, with $n=3-5$. * $P<0.05$ vs $C T R L$ and ONX-0914 (ANOVA followed by Student's $t$-test with Bonferroni correction). Results are shown as means + s.E.M.

the cytosolic NFкB subunit p65 and IкB levels (Fig. 7A, $\mathrm{B}, \mathrm{C}$ and $\mathrm{D})$. However, these cytokine-regulated changes in NFkB subunit levels were unaffected by Psmb8 inhibition (Fig. 7A, B, C and D). Since cytokine-induced IкB degradation was partial (Fig. 7D), we repeated these experiments in INS-1 in which the degradation was almost complete (Fig. 7E). In line with the results obtained from the INS-1E cells (Fig. 7D), Psmb8 inhibition did not affect cytokine-induced IкB degradation (Fig. 7E) or JNK activation (data not shown). In addition, Psmb8 inhibition did not affect IкB degradation in human islets from two donors (data not shown).

\section{Discussion}

Collectively, the data obtained in this study demonstrate that inflammatory cytokines upregulate the immunoproteasome but not the standard proteasome in human islets and the insulin-producing cell line INS-1E. The expression data (Figs 1 and 2) were remarkably similar between these systems, consistent with the highly conserved i-proteasome sequences between human and rodents (Ferrington \& Gregerson 2012). IFN $\gamma$ was the driver of i-proteasome expression (Fig. 2) with no detectable potentiation by IL-1 $\beta$. Accordingly, the Psmb8

Published by Bioscientifica Ltd. 
and Psmb9 promotors contain interferon consensus sequence 2- and gamma-activated sites that bind STAT1 and IRF-1 transcription factors (Chatterjee-Kishore et al. 2000) in addition to NFKB and AP-1 binding sequences and early growth response protein (Egr1) binding sites (James et al. 2006). The Psmb10 promoter contains similar transcription factor binding sites (Hisamatsu et al. 1996). The fact that IL-1 $\beta$ alone neither upregulated Psmb8, Psmb9 nor Psmb10 suggests that IL-1 $\beta$ upregulates Egr1, also known as Zif268 (zinc finger protein 225) or NGFI-A (nerve growth factor-induced protein A), which is known to suppress the response of the Psmb9 promoter to NFкB (James et al. 2006). Indeed, Egr1 is highly expressed in pancreatic islets and beta cells and upregulated after $4-8 \mathrm{~h}$ by cytokines in INS-1E cells in an NO-independent manner (Kutlu et al. 2003), suggesting that the cGMP/ cAMP-PKA/G-CREB pathways are not involved in regulating the beta-cell immunoproteasome in contrast to endothelial cells that have a high basal expression of the immunoproteasome.

Chymotrypsin-like activity was induced more by cytokines than caspase-like activity after $6 \mathrm{~h}$ (Fig. 3A), compatible with the fact that the Psmb9 catalytic activity to cleave after acidic amino acid residues is significantly reduced in the immunoproteasome compared to the standard proteasome (Ferrington \& Gregerson 2012) with ensuing consequences for substrate specificity. Since the assay employed does not discern between proteasomal- and non-proteasome-dependent caspase activity, it is possible that part of the cytokine-induced caspase-like activity in Fig. 3, normally almost abolished in the i-proteasome, arises from IFN $\gamma$-induced caspase 1 (Karlsen et al. 2000).

Interestingly, and in contrast to the effect of cytokines and high glucose reported here, prolonged glucotoxicity decreases proteasomal activities (Broca et al. 2014). These opposite activities may be related to the timing of the experimental conditions; we assessed the activities after just 6 and $24 \mathrm{~h}$ where Broca and coworkers (Broca et al. 2014) reported a decrease in activity after 14 days of high glucose exposure, which correlated with a substantial induction of apoptosis.

The choice of a pharmacological rather than a molecular approach to show a cause-effect relationship between i-proteasome expression and activity and islet cell damage was a deliberate strategy, since the production of a proteasome-deficiency phenotype requires triple KO of Psmb 8, 9 and 10 or double KO of Psmb9 and 10 plus inhibition with ONX 914 to inhibit Psmb8, with consequential risks of transfection artifacts. The ONX-914 inhibitor is highly selective for the i-proteasome (Muchamuel et al. 2009).

Psmb8 inhibition affected neither the activity of JNK nor the levels of p50, p52 and p65 or IкB degradation suggesting that i-proteasomal inhibition neither altered the turnover of these NFкB subunits nor NFкB pathway activity. Earlier studies demonstrated that the 26S proteasomal inhibitor MG-132 inhibited NFkB-dependent islet and insulin-producing cell iNOS and cyclooxygenase-2 (COX2) expression and activity (Kwon et al. 1998). Since NO and COX2 products are not required for cytokine-induced human islet-cell apoptosis and since we used highly selective i-proteasome inhibition, these observations are not in conflict with the data presented here. Of note, Psmb8 and Psmb9 double KO macrophages fail to activate NO production via the TRIF/ TRAF3 NFкB pathway but demonstrate unaffected Myd88/ TRAF6 NFкB-dependent TNF production, suggesting that the i-proteasome differentially regulates $\mathrm{NF \kappa B}$ signaling (Reis et al. 2011).

Taken together with our observations that Psmb8 inhibition did not affect $\mathrm{NF}_{\mathrm{K} B}$ signaling, these observations do not support NO as a mediator of the increased apoptosis observed with the i-proteasome inhibitor.

In addition to affecting inflammatory signaling, proteasome inhibition would be expected to result in reduced removal of oxidized or misfolded proteins (Seifert et al. 2010). The i-proteasome clears defective ribosomal products (DRIPS), which are misfolded or oxidized proteins produced on cytokine exposure, in particular to IFN $\gamma$ via mTOR signaling. DRIPS clearing is polyubiquitin and $19 \mathrm{~S}$ ATPase independent (Shringarpure et al. 2003). Inhibition of the i-proteasome would therefore be expected to lead to increased levels of toxic protein aggregates (aggresomes) (Seifert et al. 2010), but we were unable to detect such an effect. The observed increase in protein thiols caused by the inhibitor in the presence of cytokines may be due to increased activity of $\gamma$-glutamyl cysteine ligase, the key enzyme in synthesis of the antioxidant glutathione (Dickinson \& Forman 2002), although this protective response may be outcompeted by dominant pro-apoptotic signals.

I-proteasome deficiency or inhibition protects against dextran sodium sulfate (SDS)-induced enterocolitis and experimental arthritis in mice (Muchamuel et al. 2009, Basler et al. 2010, Schmidt et al. 2010). I-proteasome inhibition has not to our knowledge been tested in diabetic animal models, and our study clarifies the translational potential of this concept, in that our data in human islets do not support the use of inhibitors selective

Published by Bioscientifica Ltd. 
for the i-proteasome in diabetes. In conclusion, beta cells upregulate immunoproteasome expression and activity in response to IFN $\gamma$, likely as a protective response to modulate inflammatory signaling.

\section{Declaration of interest}

The authors declare that there is no conflict of interest that could be perceived as prejudicing the impartiality of the research reported.

\section{Funding}

This study was supported by the University of Copenhagen career PhD fellowship and an ELITE-research grant (to M L), CSC scholarship (to Z C), Novo Nordisk Foundation - NNF13OC0004294 (to M J D), Type 1 Diabetes Pathfinder award, NIDDK (to B K W) and Novo Nordisk Foundation (to T M P O).

\section{Author contribution statement}

$M L, M B, D H-C, B W, P M$ and $T M P$ designed the experimental setup. M L, M B, T D, D H-C, S M G, Z H and M N L performed the experiments. $M L$ and $T M P$ drafted the manuscript. M J D oversaw protein oxidation experiments and edited the manuscript. All authors approved the final version.

\section{Acknowledgements}

The authors would like to thank Francesca Grano for excellent technical assistance.

\section{References}

Basler M, Dajee M, Moll C, Groettrup M \& Kirk CJ 2010

Prevention of experimental colitis by a selective inhibitor of the immunoproteasome. Journal of Immunology 185 634-641. (doi:10.4049/jimmunol.0903182)

Bhattacharyya S, Yu H, Mim C \& Matouschek A 2014 Regulated protein turnover: snapshots of the proteasome in action. Nature Reviews: Molecular Cell Biology 15 122-133. (doi:10.1038/nrm3741)

Brand MD, Parker N, Affourtit C, Mookerjee SA \& Azzu V 2010 Mitochondrial uncoupling protein 2 in pancreatic beta-cells. Diabetes, Obesity and Metabolism 12 (Supplement 2) 134-140. (doi:10.1111/ j.1463-1326.2010.01264.x)

Broca C, Varin E, Armanet M, Tourrel-Cuzin C, Bosco D, Dalle S \& Wojtusciszyn A 2014 Proteasome dysfunction mediates high glucoseinduced apoptosis in rodent beta cells and human islets. PLOS ONE 9 e92066. (doi:10.1371/journal.pone.0092066)

Bugliani M, Liechti R, Cheon H, Suleiman M, Marselli L, Kirkpatrick C, Filipponi F, Boggi U, Xenarios I, Syed F, et al. 2013 Microarray analysis of isolated human islet transcriptome in type 2 diabetes and the role of the ubiquitin-proteasome system in pancreatic beta cell dysfunction. Molecular and Cellular Endocrinology 367 1-10. (doi:10.1016/j.mce.2012.12.001)

Chang JY \& Chang NS 2015 WWOX dysfunction induces sequential aggregation of TRAPPC6ADelta, TIAF1, tau and amyloid beta, and causes apoptosis. Cell Death Discovery 1 15003. (doi:10.1038/ cddiscovery.2015.3)
Chatterjee-Kishore M, Wright KL, Ting JP \& Stark GR 2000 How Stat 1 mediates constitutive gene expression: a complex of unphosphorylated Stat1 and IRF1 supports transcription of the LMP2 gene. EMBO Journal 19 4111-4122. (doi:10.1093/emboj/19.15.4111)

Chou DH, Holson EB, Wagner FF, Tang AJ, Maglathlin RL, Lewis TA, Schreiber SL \& Wagner BK 2012 Inhibition of histone deacetylase 3 protects beta cells from cytokine-induced apoptosis. Chemistry and Biology 19 669-673. (doi:10.1016/j.chembiol.2012.05.010)

Chou DH, Vetere A, Choudhary A, Scully SS, Schenone M, Tang A, Gomez R, Burns SM, Lundh M, Vital T, et al. 2015 Kinase-independent small-molecule inhibition of JAK-STAT signaling. Journal of the American Chemical Society 137 7929-7934. (doi:10.1021/jacs.5b04284)

Christensen DP, Gysemans C, Lundh M, Dahllof MS, Noesgaard D, Schmidt SF, Mandrup S, Birkbak N, Workman CT, Piemonti L, et al. 2014 Lysine deacetylase inhibition prevents diabetes by chromatin-independent immunoregulation and beta-cell protection. PNAS 111 1055-1059. (doi:10.1073/pnas.1320850111)

Dickinson DA \& Forman HJ 2002 Cellular glutathione and thiols metabolism. Biochemical Pharmacology 64 1019-1026. (doi:10.1016/ S0006-2952(02)01172-3)

Ferrington DA \& Gregerson DS 2012 Immunoproteasomes: structure, function, and antigen presentation. Progress in Molecular Biology and Translational Science 109 75-112. (doi:10.1016/B978-0-12-3978639.00003-1)

Hartley T, Brumell J \& Volchuk A 2009 Emerging roles for the ubiquitinproteasome system and autophagy in pancreatic beta-cells. American Journal of Physiology: Endocrinology and Metabolism 296 E1-E10. (doi:10.1152/ajpendo.90538.2008)

Hawkins CL, Morgan PE \& Davies MJ 2009 Quantification of protein modification by oxidants. Free Radical Biology and Medicine $\mathbf{4 6}$ 965-988. (doi:10.1016/j.freeradbiomed.2009.01.007)

Hisamatsu H, Shimbara N, Saito Y, Kristensen P, Hendil KB, Fujiwara T, Takahashi E, Tanahashi N, Tamura T, Ichihara A, et al. 1996 Newly identified pair of proteasomal subunits regulated reciprocally by interferon gamma. Journal of Experimental Medicine 183 1807-1816. (doi:10.1084/jem.183.4.1807)

James AB, Conway AM \& Morris BJ 2006 Regulation of the neuronal proteasome by Zif268 (Egr1). Journal of Neuroscience 26 1624-1634. (doi:10.1523/JNEUROSCI.4199-05.2006)

Kanai K, Aramata S, Katakami S, Yasuda K \& Kataoka K 2011 Proteasome activator PA28gamma stimulates degradation of GSK3phosphorylated insulin transcription activator MAFA. Journal of Molecular Endocrinology 47 119-127. (doi:10.1530/JME-11-0044)

Karlsen AE, Pavlovic D, Nielsen K, Jensen J, Andersen HU, Pociot F, Mandrup-Poulsen T, Eizirik DL \& Nerup J 2000 Interferon-gamma induces interleukin-1 converting enzyme expression in pancreatic islets by an interferon regulatory factor-1-dependent mechanism. Journal of Clinical Endocrinology and Metabolism 85 830-836. (doi:10.1210/jcem.85.2.6366)

Kitiphongspattana K, Mathews CE, Leiter EH \& Gaskins HR 2005 Proteasome inhibition alters glucose-stimulated (pro)insulin secretion and turnover in pancreatic \{beta\}-cells. Journal of Biological Chemistry 280 15727-15734. (doi:10.1074/jbc.M410876200)

Kutlu B, Cardozo AK, Darville MI, Kruhoffer M, Magnusson N, Orntoft T \& Eizirik DL 2003 Discovery of gene networks regulating cytokine-induced dysfunction and apoptosis in insulinproducing INS-1 cells. Diabetes 52 2701-2719. (doi:10.2337/ diabetes.52.11.2701)

Kwon G, Corbett JA, Hauser S, Hill JR, Turk J \& McDaniel ML 1998 Evidence for involvement of the proteasome complex (26S) and NFkappaB in IL-1beta-induced nitric oxide and prostaglandin production by rat islets and RINm5F cells. Diabetes 47 583-591. (doi:10.2337/diabetes.47.4.583)

Litwak SA, Wali JA, Pappas EG, Saadi H, Stanley WJ, Varanasi LC, Kay TW, Thomas HE \& Gurzov EN 2015 Lipotoxic stress induces pancreatic beta-cell apoptosis through modulation of $\mathrm{Bcl}-2$ proteins by the 
ubiquitin-proteasome system. Journal of Diabetes Research 2015 280615. (doi:10.1155/2015/280615)

Masini M, Bugliani M, Lupi R, del GS, Boggi U, Filipponi F, Marselli L, Masiello P \& Marchetti P 2009 Autophagy in human type 2 diabetes pancreatic beta cells. Diabetologia 52 1083-1086. (doi:10.1007/ s00125-009-1347-2)

Muchamuel T, Basler M, Aujay MA, Suzuki E, Kalim KW, Lauer C, Sylvain C, Ring ER, Shields J, Jiang J, et al. 2009 A selective inhibitor of the immunoproteasome subunit LMP7 blocks cytokine production and attenuates progression of experimental arthritis. Nature Medicine 15 781-787. (doi:10.1038/nm.1978)

Rape M \& Jentsch S 2002 Taking a bite: proteasomal protein processing. Nature Cell Biology 4 E113-E116. (doi:10.1038/ncb0502-e113)

Reis J, Hassan F, Guan XQ, Shen J, Monaco JJ, Papasian CJ, Qureshi AA, Van Way CW, Vogel SN, Morrison DC, et al. 2011 The immunoproteasomes regulate LPS-induced TRIF/TRAM signaling pathway in murine macrophages. Cell Biochemistry and Biophysics 60 119-126. (doi:10.1007/s12013-011-9183-7)

Schmidt N, Gonzalez E, Visekruna A, Kuhl AA, Loddenkemper C, Mollenkopf H, Kaufmann SH, Steinhoff U \& Joeris T 2010 Targeting the proteasome: partial inhibition of the proteasome by bortezomib or deletion of the immunosubunit LMP7 attenuates experimental colitis. Gut 59 896-906. (doi:10.1136/gut.2009.203554)

Seifert U, Bialy LP, Ebstein F, Bech-Otschir D, Voigt A, Schroter F, Prozorovski T, Lange N, Steffen J, Rieger M, et al. 2010
Immunoproteasomes preserve protein homeostasis upon interferoninduced oxidative stress. Cell 142 613-624. (doi:10.1016/j. cell.2010.07.036)

Shringarpure R, Grune T, Mehlhase J \& Davies KJ 2003 Ubiquitin conjugation is not required for the degradation of oxidized proteins by proteasome. Journal of Biological Chemistry 278 311-318. (doi:10.1074/jbc.M206279200)

Storling J, Allaman-Pillet N, Karlsen AE, Billestrup N, Bonny C \& Mandrup-Poulsen T 2005 Antitumorigenic effect of proteasome inhibitors on insulinoma cells. Endocrinology 146 1718-1726. (doi:10.1210/en.2004-0963)

Subramanian A, Tamayo P, Mootha VK, Mukherjee S, Ebert BL, Gillette MA, Paulovich A, Pomeroy SL, Golub TR, Lander ES, et al. 2005 Gene set enrichment analysis: a knowledge-based approach for interpreting genome-wide expression profiles. PNAS 10215545 15550. (doi:10.1073/pnas.0506580102)

Yamin TT \& Miller DK 1997 The interleukin-1 receptor-associated kinase is degraded by proteasomes following its phosphorylation. Journal of Biological Chemistry 272 21540-21547. (doi:10.1074/ jbc.272.34.21540)

Yan FF, Lin CW, Cartier EA \& Shyng SL 2005 Role of ubiquitinproteasome degradation pathway in biogenesis efficiency of \{beta\}-cell ATP-sensitive potassium channels. American Journal of Physiology: Cell Physiology 289 C1351-C1359. (doi:10.1152/ ajpcell.00240.2005)

Received in final form 10 April 2017

Accepted 24 April 2017

Accepted Preprint published online 24 April 2017
๑) 2017 Society for Endocrinology Printed in Great Britain
Published by Bioscientifica Ltd. 